\title{
Terahertz-Frequency Quantum Oscillator Operating in the Positive Differential Resistance Region
}

\author{
Peiji Zhao ${ }^{a}$, Dwight Woolard ${ }^{\mathrm{a}, \mathrm{b}}$, Matthew Lasater ${ }^{\mathrm{c}}$, C. T. Kelley ${ }^{\mathrm{c}}$, Robert Trew ${ }^{\mathrm{a}}$ \\ ${ }^{a}$ Department of Electrical and Computer Engineering, North Carolina State University, Raleigh, \\ NC 27696 USA \\ ${ }^{\mathrm{b}}$ US Army Research Lab., ARO, Research Triangle Park, NC, USA \\ ${ }^{c}$ Center for Research in Scientific Computation, Department of Mathematics, North Carolina State \\ University, Raleigh, NC 27696-8205, USA
}

\begin{abstract}
The traditional implementation of resonant tunneling diodes (RTD) as a high-frequency power source always requires the utilization of negative-differential resistance (NDR). However, there are inherent problems associated with effectively utilizing the two-terminal NDR gain to achieve significant levels of output power. This paper will present a new design methodology where resonant tunneling structures (RTS) are engineered to exhibit electronic instabilities within the positive-differential-resistance (PDR) region. As will be demonstrated, this approach utilizes a microscopic instability that alleviates the need to reduce device area (and therefore output power) in an effort to achieve lowfrequency stabilization
\end{abstract}

Keywords: resonant tunneling, positive differential resistance region, intrinsic high frequency oscillation

\section{INTRODUCTION}

Terahertz frequencies are defined as those in the range $100 \mathrm{GHz} \sim 10 \mathrm{THz}$ region in the electromagnetic spectrum. Terahertz frequency systems have found applications in radio astronomy [1] [2], atmospheric monitoring [3], plasma diagnostics [4], and in the study of solid state physics. There have been growing needs for terahertz electromagnetic spectrum for imaging and surveillance [5], sensing and detection in biological/chemical warfare [6], imaging of biomolecules [7], secure communication [8], and networking [9]. Though terahertz technology plays important roles both in civilian and military applications, this significant region in the electromagnetic spectrum has not been opened up for commercial exploitation. The main reason for this is the virtual absence in this frequency range of reliable, low cost, miniaturized solid-state power sources[10]. Because the terahertz frequency regime fall in between the available semiconductor lasers (which extend into the mid-infrared region) and microwave sources, the dearth of sources, detector, and interconnects for this frequency region is referred to as the famous "THz Gap" issue.

Terahertz radiation can be generated based on electronics or photonics. From the point of view of photonics, though photo-mixers are able to generate terahertz power output at several terahertz frequencies, the output power is limited to the $\mu W$ level or below [11]. While type-II quantum cascade (QC) lasers generate power output in mid-infrared range[12], type-I QC-lasers can deliver only $5 \sim 19 \mathrm{pW}$ at $2.5 \mathrm{THz}$ as reported in a recent experiment [13]. The value of this output power is too small to obtain system application. For cascade terahertz lasers, non-radiative relaxation processes forbid operation in the proximity of the materials reststrahlenbband. In addition, free carrier absorption in doped semiconductors, which scale at approximately $\lambda^{2}$, leads to increased optical losses at longer wavelengths, making QC-laser action in the terahertz region a challenging proposition [14]. From the point of view of electronics, while threeterminal devices reach higher and higher frequencies [15], two- terminal fundamental oscillators are a key technology that can open the terahertz regime [13]. Up to now, the known two-terminal power sources include impact avalanche transit-time (IMPATT) diodes, GUNN diodes, tunnel injection transit-time (TUNNETT) diodes, superlattice electronic devices (SLED), and resonant tunneling diodes (RTD). However, the output powers of these power sources in the 
terahertz frequency regime are much lower than engineering needs. Basically, most of the two-terminal devices can only provide enough power for applications at the very lower limits of the terahertz electromagnetic spectrum.

Although an RTD, designed via the most common method, can provide high frequency output at a frequency up to 712 $\mathrm{GHz}$, its output power is only $0.3 \mu \mathrm{W}[16]$. The common RTD oscillators have some inherit drawbacks. In common RTD oscillators, a RTD is utilized as a component in a traditional two-terminal oscillator source. The RTD is biased in its negative differential resistance (NDR) region. This leads to several problems severely influencing the performance of the RTD oscillators power output. First, any noise fluctuations will be amplified when the RTD and it's inherit charge storage capacitance resonates in an unstable manner with some external charge storage element (e. g., inductance of the contact lead). This effect will finally drive the balance point of the oscillation to the edge of the NDR region. Second, since a RTD acts as an unstable gain mechanism, and oscillation is produced by resonating with an external element, the energy associated with the oscillation must pass through a physical contact, which will always possess some lose. Third, the NDR of a RTD will encourage oscillation in the bias circuitry down to zero frequency. Therefore, one must design the circuit coupled to the RTD-based oscillator so that it is low-frequency stable to prevent energy losses to lower frequency modes. This requires the designer to reduce the RTD capacitance, thereby the cross-section area of the RTD. This reduction in the RTD device cross-section area severely limits the available output power of the oscillator at high frequencies.

The above research review shows that the famous "THz Gap" is still unfilled. As such, we believe that the potential and future solid state power sources that will fill the Gap have to combine the features of particle (transport based devices) and waves (wave based devices)[17][11]. The devices should be "quantum transport devices"[12]. Based on this philosophical idea, we present the following theory for the design of terahertz frequency quantum oscillators operating in the positive differential resistance region. As stated below, the oscillation is caused by the coupling of energy subbands of the system, and we call these type of oscillators quantum energy level coupling oscillators (QELCO) [18].

\section{Design Principle}

The origin of the intrinsic current oscillation in double barrier quantum well structures (DBQWS) was a long-time unsolved device physics problem[19] [21]. It was resolved only recently by the authors of the paper and their coworkers [18][22] [32]. The research results reveal the fundamental physics of quantum transport through a DBQWS. The key for understanding the origin of the intrinsic current oscillation is that the DBQWS becomes an open multisubband system once an emitter quantum well (EQW) is created in front of the emitter barrier[18][31].

\subsection{Origin of Intrinsic Current Oscillation in Multi-Subband Systems}

For an open multi-subband system, without considering the movement of carriers in a lateral direction, the wave function of the system can be written as

$$
\psi(z, t)=\sum_{k=1}^{N} \psi_{k}(z, t)=\sum_{k=1}^{N} \phi_{k}(z, t) \exp \left(-i \frac{2 \pi F_{k}(t)}{h}\right)
$$

where

$$
F_{k}(t)=\int_{0}^{t} E_{k}\left(t^{\prime}\right) d t^{\prime}
$$

$E_{k}$ is the energy of the $k t h$ energy level of the system (the energy of the $k t h$ quasi-bound state); $\psi_{k}(z, t)$ is the wavefunction of the $k t h$ quasi-bound state; $\phi_{k}$ is the amplitude of the wavefunction, a slow-varying function of $t$. With this wavefunction, the density of electrons in the structure can be written as

$$
\rho(z, t)=\sum_{k}\left|\phi_{k}(z, t)\right|^{2}+2 \operatorname{Re} \sum_{k, l(k<l)} \phi_{k}^{*}(z, t) \phi_{l}(z, t) \exp \left(-i 2 \pi \frac{F_{l}-F_{k}}{h}\right) \text {. }
$$


The oscillation terms in the above equation will usually be smeared out by the cancellation effect induced by variations in phase (e.g., unequal subband structures leading to conditions such that $E_{l_{1}}-E_{k_{1}} \neq E_{l_{2}}-E_{k_{2}}$ ) and zero coupling between the states. When these conditions apply, the transport can be described simply as an individual summation over single subbands. Hence, there is no coupling between the bands and no intrinsic oscillation. In fact, it is this type of situation where the concept of subband transport is most often applied and most useful. The time-dependent nature of the electron transport may arise due to inter-subband coupling under certain oscillation conditions. These conditions may be conveniently classified into three categories that will now be considered individually as follows:.

\section{Case 1. Maximum Subband Coherence}

If we assume that conditions sufficient for intrinsic oscillations exist, the oscillations in density will occur when all the energy-dependent phase factors in Eq. (3) are equivalent and when all contribute to the instability. This condition of maximum coherence is directly defined by the resulting subband structure and is given by the relation

$$
\Delta E_{l k}(t)=\left|E_{l}(t)-E_{k}(t)\right|=\Delta E(t) \quad \text { and } l \in\left\{l_{i}\right\}, k \in\left\{k_{j}\right\}, l<k,
$$

where the sets $\left\{l_{i}\right\}$ and $\left\{\mathcal{k}_{j}\right\}$ are of equal numbers and assume all possible values from the number sequences $1,2, \cdots, n$. It is important to note that the phase factor defined in Eq. (3) must possess a natural time dependency. This is true because any condition of oscillation in current density will be accompanied by a corresponding oscillation in the band structure of the device. In this case, the carrier density can be written as

$$
\rho(z, t)=\sum_{k}\left|\phi_{k}(z, t)\right|^{2}+2 \operatorname{Re}\left\{\exp \left(-i 2 \pi \frac{\Delta F}{h}\right) \sum_{k, l(k<l)} \phi_{k}^{*}(z, t) \phi_{l}(z, t)\right\},
$$

where $\Delta F(t)=\int_{0}^{t} d t^{\prime} \Delta E\left(t^{\prime}\right)$. The sum of all subband coupling terms (i.e. the sum in the second terms on RHS of Eq. (5) ) defines the amplitudes for oscillations in electron density.

\section{Case 2. Partial Subband Coherence}

The next level of oscillation condition is characterized by the condition where a finite and countable number of energydependent phase factors in Eq. (3) are equivalent and where each of these contributes to the instability. This condition of partial subband coherence is defined by

$$
\Delta E_{l k}(t)=\left|E_{l}(t)-E_{k}(t)\right|=\Delta E(t) \quad \text { and } \quad l \in\left\{l_{i}\right\}, k \in\left\{k_{j}\right\}, l<k,
$$

where the sets $\left\{l_{i}\right\}$ and $\left\{k_{j}\right\}$ of equal numbers with $(l<k)$ assume some of the values from the number sequences $1,2, \cdots, n$. In the case, the density of electrons can be expressed as

$$
\begin{aligned}
\rho(z, t) & =\sum_{k}\left|\phi_{k}(z, t)\right|^{2}+2 \operatorname{Re}\left\{\exp \left(-i 2 \pi \frac{\Delta F}{h}\right) \sum_{\left.k, l(k<l), t \in l_{i}\right\}, k \in\left\{k_{j}\right\}} \phi_{i}^{*}(z, t) \phi_{l}(z, t)\right\} \\
& +2 \operatorname{Re}\left\{\sum_{k, l(k<l),\left\{\notin l_{i}\right\}, k \notin\left\{k_{j}\right\}} \phi_{k}^{*}(z, t) \phi_{l}(z, t) \exp \left(-i 2 \pi \frac{F_{l}(t)-F_{k}(t)}{h}\right)\right\} .
\end{aligned}
$$

Under these conditions, the in-phase contributions from only the second terms on the RHS of Eq. (7) will contribute to the intrinsic oscillations as the last terms lack sufficient coherence.

Case 3. Minimum Subband Coherence 
The last and weakest form of intrinsic oscillations is characterized by the condition where only a single set of subbands contributes to the instability. Assuming that the $l_{i}$ and $k_{j}$ subbands contribute to the oscillation where $l_{i}$ and $k_{j}$ are the only subband indices that contribute to the oscillation in the energy level index $1,2, \cdots, n$. Thus, the density of the electrons can be written as

$$
\begin{aligned}
\rho(z, t) & =\sum_{k}\left|\phi_{k}(z, t)\right|^{2}+2 \operatorname{Re}\left\{\exp \left(-i 2 \pi \frac{\Delta F}{h}\right) \phi_{k_{j}}^{*}(z, t) \phi_{l_{i}}(z, t)\right\} \\
& +2 \operatorname{Re}\left\{\sum_{k, l(k<l), l \neq l_{i}, k \neq k_{j}} \phi_{k}^{*}(z, t) \phi_{l}(z, t) \exp \left(-i 2 \pi \frac{F_{l}(t)-F_{k}(t)}{h}\right)\right\} .
\end{aligned}
$$

As in the prior case, the in-phase contributions from only the second terms on the RHS of Eq. (8) will contribute to the intrinsic oscillations.

For each of the three previous subband coherence cases, the electron density can be expressed most generally as

$$
\rho(z, t)=\sum_{k}\left|\phi_{k}(z, t)\right|^{2}+2 \operatorname{Re}\left\{\exp \left(-i 2 \pi \frac{\Delta F}{h}\right) G(t)\right\},
$$

where the functions $G$ are slowly-varying functions of time. It accounts for the contribution to the charge density from the coupling between the subbands. Here, the incoherent subband terms in Eq. (1) have been excluded. Also while the first terms have been retained, it should be noted that they only contribute to short term transients and to the final static components of electron density[23][27]. The expression defined in Eq. (9) reveals that intrinsic high frequency oscillations can arise in any quantum system from the wavefunction coupling between multi-subbands. The oscillations occur once the subbands structure satisfies one of the criteria given in Eqs.(4) (8) and the inter-subband coupling function $\mathrm{G}$ is not equal to zero. These criteria and the non-zero-coupling condition $(G \neq 0)$ set up the fundamental conditions that a multi-subband semiconductor system has to be satisfied if the intrinsic current oscillations exist in the system. Further analysis of the equations can provide a clear physical picture regarding the creation of the oscillation. The key is the energy-dependent phase factors in Eq.(9). It should be noted that prior simulations have shown that the self-consistent potential varies in a periodical form [23]. Thus, it is reasonable to express the energy difference between the energy subbands as

$$
\Delta E(t)=\Delta E_{0}+f(\omega, t)
$$

where $f(\omega, t)$ is a periodical function and $\omega$ is the oscillation frequency. $\Delta E_{0}$ is defined as the average energy difference between two energy levels for a system subject to intrinsic oscillations, or the energy difference at the balance point. Obviously, the phase difference in Eq.(9) between time $t_{1}$ and $t_{2}$ can be written as

$$
\Delta \varphi\left(t_{1}, t_{2}\right)=2 \pi \frac{\Delta E_{0}}{h}\left(t_{1}-t_{2}\right)+\frac{2 \pi}{h} \int_{t_{1}}^{t_{2}} f\left(\omega, t^{\prime}\right) d t^{\prime} .
$$

Recognizing that the phase variation in one period is $2 \pi$ and the periodicity of the function $f(\omega, t)$, the oscillation frequency of the current, due to the subband structure, is given by

$$
\frac{1}{T}=\frac{\Delta E_{0}}{h}
$$

where $T$ is the period of the intrinsic oscillation. The previous derivation allows us to establish a physics-based description for the creation of the intrinsic oscillation. The accurate physical model for this instability process will be able to describe the time-dependent variations in electron density and potential energy. Consider for example an arbitrary oscillation process and assume that the density of electrons at a particular real-space point reaches its maximum value at $t_{0}$. The corresponding potential energy at this same space point will also assume its maximum value at time $t_{0}$. This 
statement is true at least at the level of Hartree approximation to electron-electron interactions. Assuming an oscillation condition exists, this variation in both electron density and electron potential energy will cycle periodically as the phase varies over $2 \pi$. Eq.(9) for electron density directly exhibits this type of behavior through the energy-dependent phase factors. In turn, this variation of density in time would impose variations in the potential energy. It should be noted that the periodical variation of potential energy in time definitely influences the energies of the subbands of the systems. This process also definitely leads to the oscillation of the current because of the periodical variation of the subband energies of the systems. This is the key mechanism leading to the occurrence of intrinsic current oscillations in multi-subband systems.

This quantum-based model allows one to analyze the intrinsic oscillation process to determine the underlying physical mechanisms responsible for the instability. Specifically, if detailed simulations are utilized to derive energies of the subbands and the appropriate $\Delta E_{0}$ under the condition of intrinsic oscillation, then insights into the fundamental physical mechanism response for the instability can be obtained. The next two subsections of this paper will present simulation tools and studies that allow for a complete analysis of the intrinsic oscillations in DBQWSs. In particular, Wigner-Possion Equations simulations and Schrödinger-based simulations will be used together to derive current density oscillations, the subband energy differences, and the subband wavefunction amplitudes. This information will be combined with the previous model to reveal the fundamental origins of intrinsic oscillations.

\section{NUMERICAL EXPERIMENT VERIFICATION OF THE DESIGN THEORY}

In this subsection, numerical simulation methods will be used against a heterostructure system to verify the multisubband theory for the design of a QELCO. In order to incorporate the dissipation effects (electron-phonon interactions), the Wigner-Poisson device model, that is well accepted, will be used to model the quantum devices. The resulting selfconsistent potential will be used as an input for solving the Schroedinger equation to get the energy level structure so that the oscillation frequency can be calculated by using Eq.(11). The calculated frequency can then be used to compare with the Wigner-Poisson result to verify the theory.

\section{a). Wigner-Poisson Model of Quantum Devices}

The Wigner function formulation of quantum mechanics was selected for this study because of its many useful characteristics for the simulation of quantum-effect electronic devices, including the natural ability to handle dissipate and open-boundary systems. The Wigner function equation was first employed in quantum device simulation by Frensley [33]. Later, Kluksdahl et al incorporated Poisson's equation (PE) and applied the model to the study of RTD's with self-consistent method [34]. With the lowest order approximation to scattering being considered only, we have

$$
\frac{\partial f}{\partial t}=-\frac{h k}{2 \pi m^{*}} \frac{\partial f}{\partial x}-\frac{1}{h} \int d k^{\prime} f\left(x, k^{\prime}\right) \int d y\{U(x+y)-U(x-y)\} \sin \left(2 y\left(k-k^{\prime}\right)\right)+\left.\frac{\partial f}{\partial t}\right|_{\text {coll }},
$$

where $\mathrm{h}$ is the Planck's constant, $m^{*}$ is the electron effective mass and $U$ is the conduction-band-edge. Here, $U=u+\Delta V, u$ is the potential energy, and $\Delta V$ is the band offset of a heterostructure system. An appropriate treatment of scattering in semiconductors is very important for getting accurate transport results. Recent research has shown that the computation burden associated with a detailed consideration of electron-phonon scattering is very formidable[35]. This huge amount of computation time would severely impede a study such as the one presented here if the electron scattering was modeled from first principles. Thus, the relaxation time approximation to scattering has been employed. The relaxation time approximation is an well-accepted approximation to scattering in numerical calculations. In terms of the relaxation time approximation to scattering, the collision terms in the above equation can be written as

$$
\left.\frac{\partial f}{\partial t}\right|_{\text {coll }}=\frac{1}{\tau}\left\{\frac{f_{0}(z, k)}{\int d k^{\prime} f_{0}\left(z, k^{\prime}\right)} \int d k^{\prime} f\left(z, k^{\prime}\right)-f(z, k)\right\}
$$

where $\tau$ is the relaxation time and $f_{0}$ is the equilibrium Wigner function. The device under study is subject to open boundaries at the emitter and collector, hence, the boundary conditions for the Wigner function equation are 


$$
\begin{aligned}
& \left.f\right|_{x=0, k>0}=\frac{4 \pi m^{*} k_{b} T}{h^{2}} \ln \left\{1+\exp \left[-\frac{1}{k_{b} T}\left(\frac{h^{2} k^{2}}{8 \pi^{2} m^{*}}-\mu_{0}\right)\right]\right\}, \\
& \left.f\right|_{x=L, k<0}=\frac{4 \pi m^{*} k_{b} T}{h^{2}} \ln \left\{1+\exp \left[-\frac{1}{k_{b} T}\left(\frac{h^{2} k^{2}}{8 \pi^{2} m^{*}}-\mu_{L}\right)\right]\right\} .
\end{aligned}
$$

The second equation used in the RTD model is the Poisson

$$
\frac{d^{2}}{d x^{2}} u(z)=\frac{q^{2}}{\varepsilon}\left[N_{d}(z)-n(z)\right]
$$

where $\varepsilon$ is the dielectric permitivity, $u(z)$ is the electrostatic potential, $q$ is the electronic charge, $N_{d}$ is the concentration of ionized dopants, and $n(z)$ is the density of electrons, given by

$$
n(z)=\int_{-\infty}^{\infty} \frac{d k}{2 \pi} f(z, k) .
$$

The current density is written as

$$
j(z)=\int_{-\infty}^{\infty} \frac{d k}{2 \pi} \frac{h k}{2 \pi m^{*}} f(z, k) .
$$

\section{b). Determination of Subband Energies}

For the quasibound states, the wavefunctions of the states can be written as

$$
\psi_{k}(z, t)=\phi_{k}(z, t) e^{-\frac{2 \pi i}{h} F_{k}(t)}, \quad k=1,2, \ldots, n
$$

where $F_{k}(t)$ is defined by Eq.(2). Substituting this wavefunction into time-dependent Schrödinger equation, we can, since we have assume that the amplitude of the wavefunction is a slow variation function of time, got the timeindependent like Schrödinger equation as the follow

$$
\hat{H}(z, t) \psi_{k}(z, t)=E_{k}(t) \psi_{k}(z, t),
$$

where $\hat{H}$ is single particle Hamiltonian which is given by

$$
\hat{H}(z, t)=-\frac{h^{2}}{(2 \pi)^{2} m^{*}} \frac{\partial^{2}}{\partial x^{2}}+V(z, t) .
$$

Here, $V(z, t)$ is potential. Pre-research shows that the amplitude of the potential is small [24]. For quasi-bound states, the boundary conditions can be written as

$$
\left.\psi_{k}\right|_{z \rightarrow-\infty}=0
$$

where we have defined $z=0$ as the boundary between the reservoir and the emitter of a device. In Eq.(20), the potential profile of the device is taken from the Wigner-Poisson simulation result. Since the potential profile is complicated, the Schroedinger equation can only be solved by numerical method.

By numerically solving the Schrödinger equation, one can calculate the energy difference between subbands. The energy differences between subbands are time-dependent and oscillatory if the current oscillates. By setting the oscillatory part of the energy difference to zero, we can get $\Delta E_{0}$. Thus, the oscillatory frequency of the current can be calculated by using Eq.(11). Comparing the frequency calculated by using Eq.(11) and the frequency from the simulated results by 
solving the Wigner-Poisson equations, we are able to verify the correctness of our design theory of a QELCO and the theory on the origin of intrinsic current oscillation through a quantum system.

\section{c). Simulation Results and Verification}

According to the design theory of a QELCO, the current oscillation in a multi-subband system exists as long as the subband energy difference satisfies one of the three criteria and the non-zero-coupling condition stated in Section 2.2.1. In order to verify the QELCO concept provided above, a double barrier quantum well structure (DQWES) as shown in Fig. 2 is employed in the numerical simulation. This structure has been used by many researchers to show the current instability in a double barrier quantum well structure[20][36]. The parameters used in our simulation are the following. Momentum and position space is broken into 72 and 86 points, respectively. The donor density is $2 \times 10^{18}$ particles $/ \mathrm{cm}^{3}$; the compensation ration for scattering calculations is 0.3 ; the barrier and well widths are 30 and $50 \AA$, respectively; the simulation box is $550 \AA$; the barrier potential is $0.3 \mathrm{eV}$, corresponding to $A l_{0.3} G a_{0.7} A s$; the device temperature is $77 \mathrm{~K}$ except that we point out; the effective mass of electron is assumed to be a constant and equals to $0.0667 m_{0}$; the doping extend to $30 \AA$ before the emitter barrier and after the collector barrier; the quantum-well region is undoped. Bulk GaAs parameters are used to calculate the relaxation time and the chemical potential. The chemical potential is determined by $\int_{0}^{\infty} d \varepsilon \sqrt{\varepsilon} f(\varepsilon)=\frac{2}{3} \mu(T=0)^{\frac{3}{2}}$, where $f(\varepsilon)$ is the Fermi distribution function.

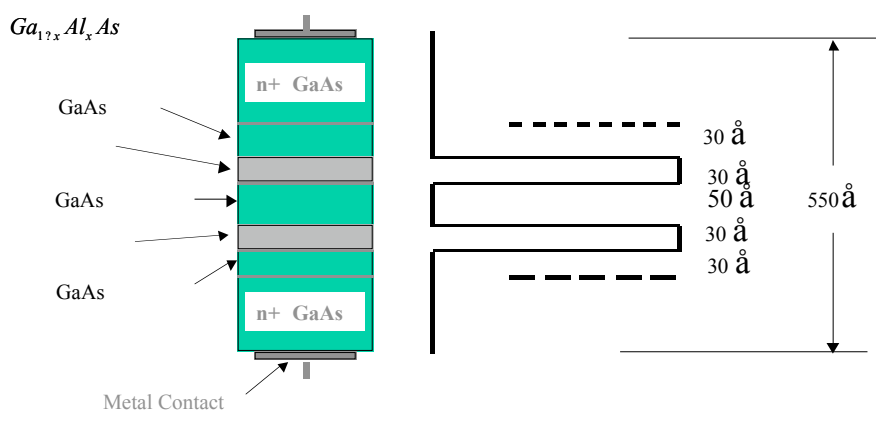

Fig. 1 Geometric structure of the device used in the simulation.

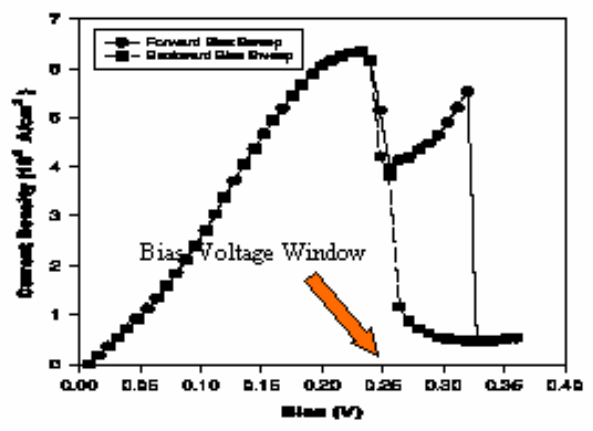

Fig. 2 I-V characteristics of the double barrier quantum well structure. The bias voltage window is in the range from $0.240 \mathrm{~V}$ to $0.248 \mathrm{~V}$. The value of the current density in the bias voltage window in this figure is the time-average of the time-dependent current density 
Fig.2 shows the I-V characteristics of the double barrier quantum well structure (DBQWS) used in the simulation. It reproduces the main features of the experimental results, thereby verifying the correctness of our simulation [23]. In the simulation, we find a bias voltage window in which the current oscillates is shown in Fig.2 and Fig.3. The oscillation frequency is $2.8 \mathrm{THz}$. The current oscillation is intrinsic. Being a long-time unsolved problem, the origin of the intrinsic current oscillations has been explained by the dynamic creation of an EQW in front of the emitter barrier [18] [23] and the theory mentioned above in the paper. We have presented a detailed explanation on why there are no current oscillations outside of the bias voltage window [18][23][25][29][31]. The cause for no current oscillation outside of the bias voltage window is that the subband structures do not satisfy the oscillation criteria and the non-zero-coupling

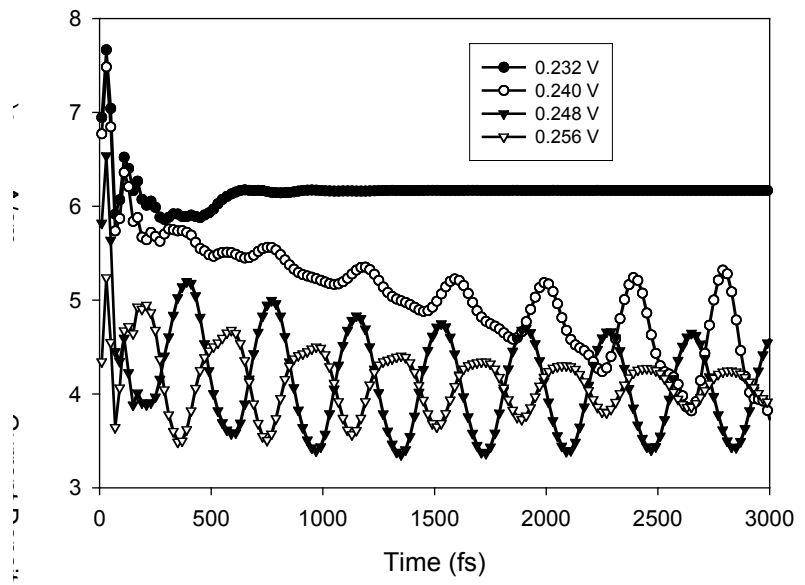

Fig. 3 Time-dependent current density at bias voltages in the bias voltage widow $(0.240 \mathrm{~V} \sim 0.248 \mathrm{~V})$.

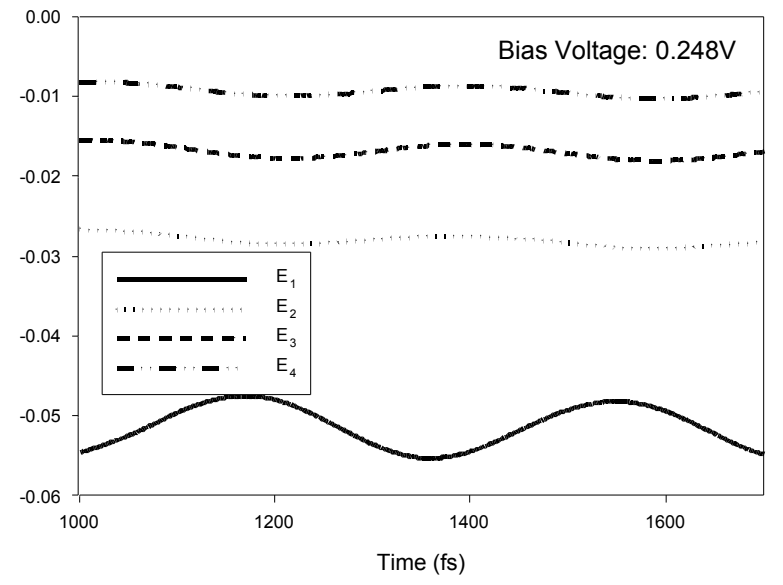

Fig.4 Time-dependent of the energy level structure of the double barrier quantum well system defined in Fig 1

condition. Here, in order to save space, we consider only the case in the bias voltage window. Fig.4 shows the energy level structure of the system at bias voltage $0.248 \mathrm{~V}$. From this figure we can see the following facts: a). the distance

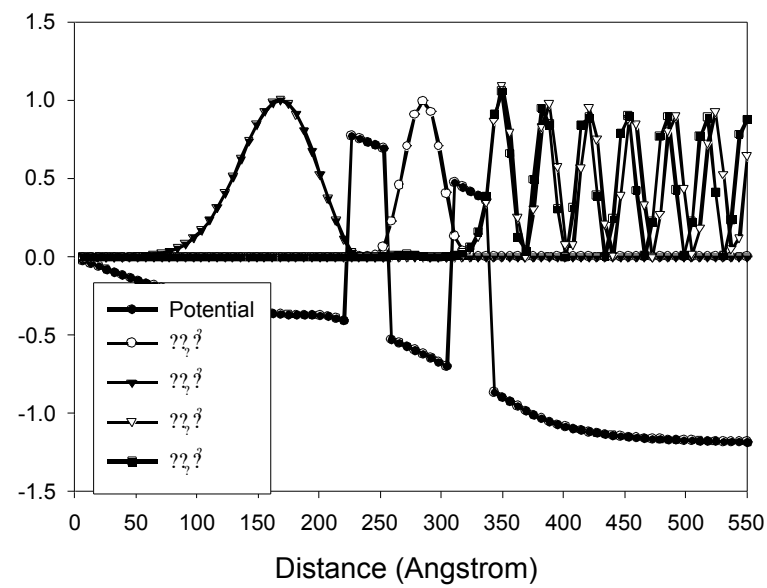

Fig.5 Normalized square of the wavefunctions at $0.248 \mathrm{~V}$ and simulation time $2500 \mathrm{fs}$. This figure shows only the states $\phi_{2}$ and $\phi_{3}$ are sited in the same spatial region. Thus, only these two states satisfy the non-zero-coupling condition. The potential profile shows the position of the well. The unit of the potential is arbitrary. between $E_{1}$ and $E_{2}$ is big. This lead to the small coupling strength between the two energy levels. Fig. 5 clearly verifies this analysis since these two states locate in different spatial regions. b). The numerical calculation shows that the width of $E_{1}$ is in the order of magnitude of $10^{-4} \mathrm{eV}$. The width of $E_{4}$ is in the order of magnitude of $10^{-7} \mathrm{eV}$. Thus, the contributions from these two energy levels to the current oscillations will disappear with the increase of the time. c) Fig. 6 shows that the coupling between $E_{1}$ and $E_{2}, E_{2}$ and $E_{4}, E_{1}$ and $E_{3}$, and $E_{3}$ and $E_{4}$ are very small, respectively. Only $E_{2}$ and $E_{3}$ have non-zero-coupling. Numerical calculation shows that the widths of $E_{2}$ and $E_{3}$ are in the order of magnitude of $10^{-40} \mathrm{eV}$. These facts show that only two energies $E_{2}$ and $E_{3}$ can contribute to the current oscillations. Numerical calculation shows that $\Delta E_{0}=$ $11.07 \mathrm{meV}$ for $E_{2}$ and $E_{3}$. Substituting this value of 
$\Delta E_{0}$ into Eq.(11), we can get the current oscillation frequency 2.68THz. Fig.3 shows that the numerical simulation result of the current oscillation frequency (from Wigner-Poisson model) is $2.8 \mathrm{THz}$. Hence, the theory presented in Section 2.2.1 of this paper provides an excellent explanation to the numerical experiment of intrinsic current oscillation in a double barrier quantum well system.

\section{NEW DEVICE STRUCTURE AND ANALYSIS}

Although our numerical experiment clearly shows the existence of intrinsic current oscillations at terahertz frequency in a multi-subband system, the bias voltage window in which the current oscillates for the device structure described above sits in the negative differential resistance region. As stated previously, current oscillations in negative differential resistance regions will severally limit the value of the output power of power sources since the area of the cross-section of the device has to be small. In order to overcome this engineering problem in design of the DBQWS-based teraherz oscillator, a shallow well is "built" just in front of the emitter barrier, as shown in Fig.6. This shallow well changes the condition that a deeper and wider EQW can be created, so that the criteria for the creation of the intrinsic current oscillation in the DBQWS can be realized at bias voltages outside of the negative resistance region. In terms of this design skill, the I-V characteristics of the device is changed. The bias voltage window is moved out of the negative differential resistance region. Fig.7 and Fig.8 show the I-V characteristics and the time-dependent current in the new bias voltage window, respectively

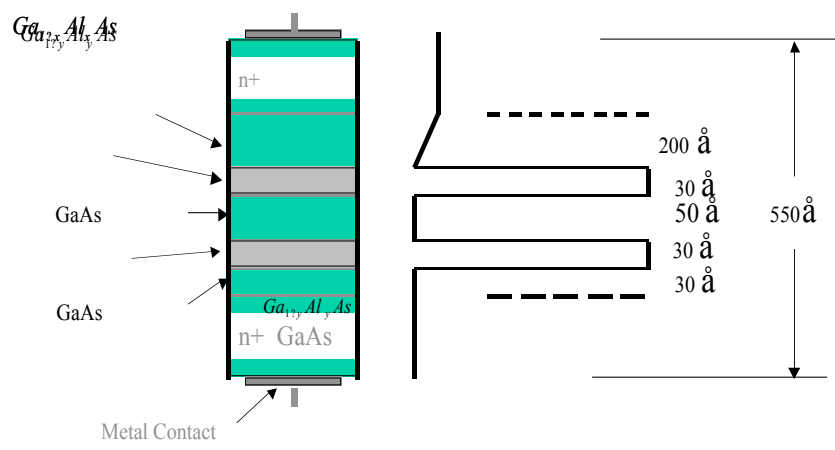

Fig. 6 A QELCO device structure and conduction band profile illustration.

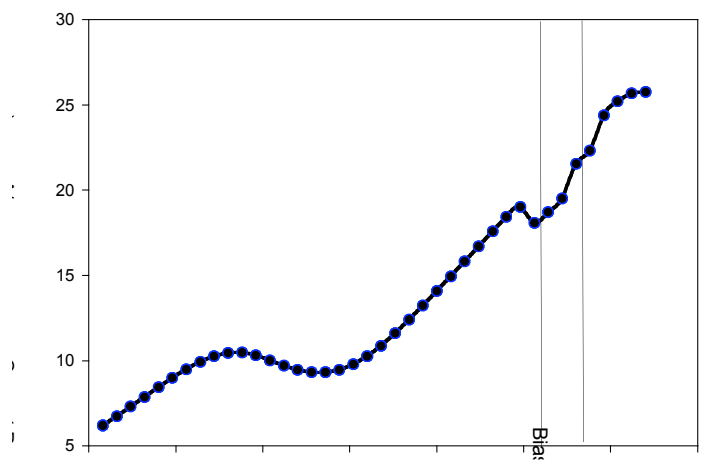

Fig. 7. I-V characteristics of the new device structure. In this bias voltage window, the current density oscillates. The values of the current density in the bias voltage window in this figure is the timeaverage of the time-dependent current density.

Fig.7 and Fig.8 show the salient features of the suggested terahertz power source, the QELCO. These features can be summarized as follows.

- The bias voltage window in which the current oscillates is located in positive differential resistance region. Thus, the area of the cross-section of the device can be larger. Because of this, the QELCO can produce larger output power.

- The output power per unit area is large. From classical Electromagnetism, the average output power can be approximately written as [37]

$$
S_{a v}=\frac{1}{2} E_{a}^{2} \operatorname{Re} \frac{1}{Z_{0}},
$$

where $Z_{0}$ is the intrinsic impedance of the material and $E_{a}$ is the amplitude of the electric field. Without considering the reflection from the load (considering an ideal case), $Z_{0}=126 \Omega$ for GaAs. The electric 
field can be estimated by

$$
E=\frac{m^{*}}{e^{2} n} \frac{\partial j(t)}{\partial t}
$$

where $m^{*}$ is the effective mass of electrons; $\mathrm{n}$ is the density of the electron. The current density shown in Fig. 8 can be approximately expressed as

$$
j(t)=1.9 \times 10^{6}-A_{1} \cos (2 \pi v t),
$$

where $A_{1}=2.5 \times 10^{5} \mathrm{~A} / \mathrm{cm}^{2}, v=3.57 \mathrm{THz}$. Thus, we got

$$
S_{a v}=\frac{1}{2}\left(\frac{m^{*} \omega A_{1}}{e^{2} n}\right)^{2} \operatorname{Re} \frac{1}{Z_{0}}=0.22 \mathrm{~W} / \mu \mathrm{m}^{2} .
$$

It should be noted that this value of the output power is larger than the expected experimental value. This is because the model used in the pre-research is one-dimensional. In fact, the lateral motion of electrons will reduce the value of the current density, thereby reducing the output power. Regardless, this value of the output power is the largest theoretical value estimated by a one-dimensional model in terahertz frequency range known. Separated estimation shows that the output power should be at least in the order of several $\mathrm{mW}[39]$.

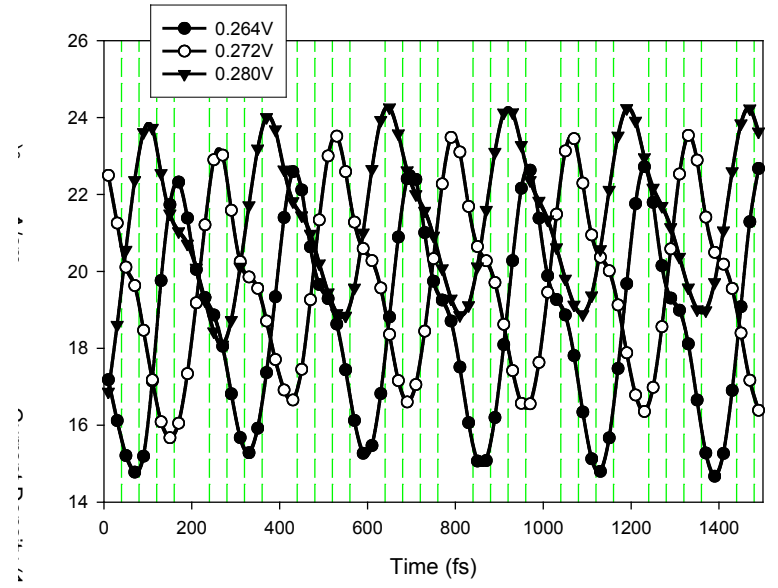

Fig.8 Time-dependent current density in the bias voltage window. The oscillation frequency is tunable. For the structure, the frequency tuning region is $3.57 \mathrm{THz} \sim 3.85 \mathrm{THz}$.

- The current oscillation is intrinsic, independent of the external circuit. This feature provides conveniences to designers of the QELCO.

- The operation temperature of the QELCO is in the temperature region of liquid nitrogen. In contrast, QC lasers can provide terahertz power output in the order of pico-watts only at very low temperature, such as $5 K^{0}$.

- The output frequency of the QELCO is electrically tunable since the subband structure of the system is tunable with the tuning of the bias voltages. For the device structure shown in Fig.7, the output frequency is tunable in the range of $3.57 \sim 3.85 \mathrm{THz}$. In the simulation of another device structure, a frequency tuning range $2.5 \sim 3.3 \mathrm{THz}$ has been found. Thus, the frequency tuning range can be enlarged by carefully designing the device structure.

\section{CONCLUSION}

In this paper, we presented a design theory of a terahertz frequency quantum oscillator operating in the positive differential resistance region. The operational principle of the oscillator is based on quantum interference between the injected electron wave and the reflected electron wave leading to the depletion of the charges in part of the region in 
front of the emitter barrier. The action of electron waves further leads to the creation of an emitter quantum well. Following the creation of the emitter quantum well, the coupling between the energy subbands leads to the current oscillations in the structure. Our simulation shows that a small well in front of the emitter barrier can change the creation condition of the emitter quantum well. This design technique presented successfully leads to instability behavior located within the positive differential resistance region. Analysis shows that the QELCO operating in a positive differential resistance region has the potential of providing more output power than other high frequency power sources in the terahertz frequency domain.

Support by the DURINT grant is acknowledged.

\section{REFERENCES}

1. http://www.mtinstruments.com/news.htm

2. T. G. Phillips and J. Keene, Submillimeter Astronomy, Proc. IEEE, 80, 1662(1992).

3. J. W. Waters, Microwave Limb Sounding, in M. A. Janssen (ed.) Atmospheric Remote Sensing, John Wiley and Sons Inc., New York, pp. 383 (1993).

4. P. E. Stott, G. Gorina, and E. Sindoni, Diagnostics for Experimental Thermonuclear Fusion Reactors, Plenum Press, New York.

5. B. B. Hu and M. C. Nuss, Optics Letters 20, 1716(1995).

6. See Army Research Office SBIR research oscillation, 2002.

7. A. G. Markelz, A. Roitberg, and E. J. Heilweil, Palsed terahertz spectroscopy of DNA, Bovine serum albumin and collagen between 0.1 and 2.0THz, Chem. Phys. Lett., 320, 42(2000).

8. N. J. Cronin, Phil. Trans. R. Soc. Lond. A354, 2425(1996).

9. Y. Takimoto, Recent activities on millimeter-wave indoor LAN system development, IEEE NTC' 95- The Microwave Systems Conference, Orlando, pp7-10; H. H. Meinel, Commercial Applications of Millimeter Waves: History, Present Status and Future Trends, IEEE Trans. MTT. 43, 1639(1995).

10. J. M. Chamberlain, R. E. Miles, C. E. Collins, and D. P. Steenson, Introduction to Terahertz Solid-State Devices, in New Directions in Terahertz Technology, edited by J. M. Chamberlain and R. E. Miles, NATO ASI Series, Vol. 334, Kluwer Academic Publishers, 1997.

11. S. J. Allen, J. S. Scott, M.C. Wanke, K. Maranowski, A. C. Gossard, M. J. W. Rodwell, and D. H. Chow, Terahertz dynamics in Quantum Structures: Towards a Fundamental Terahertz Oscillator, in Terahertz Sources and Systems, edited by R. E. Miles, P. Harrison, and D. Lippens, Kluwer Academic Publishers, 2001

12. R. Colombelli, A. Straub, F. Capasso, C. Gmachi, M. I. Blakey, A. M. Sergent, S. N. G. Chu, K. W. West, and L. N. Pfeiffer, Terahertz electroluminescence from superlattice quantum cascade structures, J. Appl. Phys., 91, 3526(2002).

13. R. Q. Yang, J. L. Bradshaw, J. D. Bruno, J. T. Pham, and D. E. Wortman, IEEE. J. Quantum Electron, 37, 282(2001).

14. A. Tredicucci, C. Gmachi, M. C. Wanke, F. Capasso, A.L. Hutchinson, D. L. Sivco, S. -N. G. Chu, and A. Y. Cho, Superlattice QC lasers towards the far-infrared, in Terahertz Sources and Systems, edited by R. E. Miles, P. Harrison, and D. Lippens, Kluwer Academic Publishers, 2001

15. M. S. Shur and V. Ryzhii, Emerging Solid State Terahertz Electronics, in Terahertz Sources and Systems, edited by R. E. Miles, P. Harrison, and D. Lippens, Kluwer Academic Publishers, 2001

16. E. R. Brown, J. R. Soderstron, C. D. Parker, L. J. Mahoneg, K. M. Molvar, and T. C. McGill, Oscillation up to $712 \mathrm{GHz}$ in InAs/AlSb resonant tunneling diodes, Appl. Phys. Lett., 58(20), 2291(1991).

17. Peiji Zhao, Origin of Intrinsic Current in Double Barrier Quantum Well Systems, Seminar, Stevens Institute of Technology, May, 2000.

18. Peiji Zhao and D. Woolard, pending US patent, A multi-subband theory for design of THz oscillators.

19. B. Ricco and M. Ya. Azbel, Phys. Rev. B29, 1970(1984).

20. K. L. Jensen and F. A. Buot, Phys. Rev. Lett. 66, 1078(1991).

21. D. L. Woolard, et al, IEEE Trans ED, 43, 332(1996).

22. Peiji. Zhao, H. L. Cui, and D.L. Woolard, K. L. Jensen, and F. A. Buot, Origin of the intrinsic bistability and plateau-like behavior of the I-V characteristics of resonant tunneling structures, J. Appl. Phys. , 87, 1337(2000).

23. Peiji Zhao, H. L. Cui and D. Woolard, Dynamical Instabilities and I-V Characteristics in Resonant Tunneling through Double Barrier Quantum Well Systems, Physical Review B63, 75302(2001). 
24. Peiji Zhao; Hong Liang Cui; Woolard, D.L.; Jensen, K.L.; Buot, F.A., Equivalent circuit parameters of resonant tunneling diodes extracted from self-consistent Wigner-Poisson simulation, IEEE Transactions on Electron Devices, Vol. 48 Issue: 4 , April 2001 Page(s): 614 -627.

25. FA Buot, Peiji Zhao, HL Cui, DL Woolard, KL Jensen, CM Krowne, Emitter quantization and double hysteresis in resonant tunneling structures: A nonlinear model of charge oscillation and current bistability, Phys. Rev. B61, 5644(2000).

26. D. Woolard, Peiji Zhao, and H. L. Cui, THz-Frequency Intrinsic Oscillations in Double-Barrier Quantum Well Systems. HCIS-12, Sante Fe, New Mexico, USA, 2001; Physica B, Vol.314, Nos.1-4, pp.108-112,2002.

27. Peiji Zhao, H.L. Cui, and D. Woolard, Design principle of resonant tunneling THz oscillator at fixed bias voltages Computational Electronics, 2000. Book of Abstracts. IWCE Glasgow 2000. 7th International Workshop on , 2000 ,Page(s): 146 -147; VLSI Design, Vol.13, Nos. 1-4, pp413-417(2001).

28. Peiji Zhao, H. L. Cui, Operational Principle of an intrinsic designed THz oscillator, presentation in International THz Symposium 2000, Michigan, May 2000

29. D. Woolard, Peiji Zhao, and H. L. Cui, Engineering Design Technique for Creating Intrinsic Current Oscillation in Double Barrier Quantum Well Systems, Second IEEE Conference on Nano-technology, Washington DC 2002.

30. Peiji Zhao, D. Woolard, and H. L. Cui, Creation and Disappearance Mechanism of the Emitter Quantum Well of a Resonant Tunneling Structure, J. Appl. Phys. 94, 1833 (2003).

31. Peiji Zhao, D. Woolard, and H. L. Cui, A Multi-Subband Theory for the Origination of Intrinsic Oscillations within Double-Barrier Quantum-Well Systems, KB-II, German, 2002; Phys. Rev. B67, 085312(2003).

32. Peiji Zhao, with D. Woolard, et al, Chapter 1, Advanced theoretical techniques for study of instability processes in nanostructures, in Advance in THz Technology, World Scientific, 2003

33. W. R. Frensley, Rev. Mod. Phys., vol. 36, 1570(1987).

34. Kluksdahl et al, Phys. Rev. B39, 7720(1988).

35. P. Bordone, M. Pascoli, R. Brunetti, A. Bertoni, and C. Jacoboni, Phys. Rev.

B59, 3060(1999).

36. B. A. Biegel and J. D. Plummer, Phys. Rev. B54, 8070(1996).

37. John D. Kraus and Daniel A. Fleisch, Electromagnetics with Application, fifth edition, McGraw-Hill, 1999.

38. T. Figielski, et al, Effect of the Device Size on the Performance of Resonant Tunneling Diodes, Proceedings EDMO, Vienna, 2001.

39. D. L. Woolard, et al, 53rd Annual Device Research Conference Digest 1995, p54. 